\title{
Zauberwort "Promotion"
}

\section{Zur Situation der katholischen Kirchenpresse in den USA}

Die Überschrift beschönigt nichts: "Circulation figures down in 1996" "Auflagenzahlen 1996 rückläufig". Die Unterzeile des Berichts über die Entwicklung der katholischen Presse in den USA, der in der FebruarAusgabe 1996 des Informationsblattes der Catholic Press Association (CPA), "The Catholic Journalist", erschienen ist, differenziert zwar: "Auflage der Bistumszeitungen stabil - kann aber Verluste der anderen Zeitschriften nicht aufwiegen". In den USA sind seit etwa drei Jahren die unbeschwerten Jahre einer blühenden Presse der Kirche vorbei.

Die Gesamtauflage der fast 650 Titel ist im vergangenen Jahr um 1,1 Mio. gesunken, das sind über $4 \%$ von 26,5 Mio. (womit das Verhältnis von 26 Mio. Auflage zu 60 Mio. Katholiken immer noch viel besser ist als in Deutschland mit 8 Mio. Auflage der Kirchenpresse zu 35 Mio. Katholiken - selbst wenn man in den USA etwa 3,7 Mio. Auflage der Newsletter abzieht, weil ähnlich geartete Mitteilungsblätter in Deutschland nicht zur Pressestatistik gerechnet werden). Die größten Verluste haben in den USA die überregionalen Zeitungen mit $10 \%$ erlitten (dazu zählen sowohl der als fortschrittlich geltende "National Catholic Reporter" als auch das traditionelle Familienblatt „Our Sunday Visitor"). Stark verloren haben mit über 7\% auch die Magazine, darunter die Zeitschriften der Orden und Missionsgesellschaften. Gehalten haben sich 1995 die Newsletters und auch die Bistumszeitungen, die 1993 und 1994 größere Einbrüche hinnehmen mußten.

Inzwischen haben in den USA 167 der 180 Diözesen eigene Bistumszeitungen, die allerdings in Format, Umfang und inhaltlicher Gestaltung (z.T. auch Erscheinungsweise: einige nur zweiwöchentlich) noch stärker variieren als die deutsche Bistumspresse. Grundverschieden vom deutschen Vertriebssystem des bezahlten Einzelbezugs ist in den USA der Vertrieb über die Pfarreien; diese erhalten von den Verlagen oder bestellen bei ihnen soviele Exemplare, wie sie brauchen und bekommen diese auch in Rechnung gestellt. Die meisten Gemeinden verkaufen die Blätter zum normalen Bezugspreis weiter an die Abonnenten, gewähren aber oft auch Preisnachlässe oder liefern sogar kostenlos. Die meisten Bischöfe unterstüzen dabei einen sog. Full coverage-Plan, wonach die Pfarrgemeinden gehalten sind, in jeden katholischen Haushalt eine Bistumszeitung zu vermitteln.

Allerdings gehört dieses Vertriebssystem zu den beiden Schwachstellen in der gegenwärtigen Situation der US-Kirchenpresse. Pfarrer und Gemeinden stehen der "Zwangsbelieferung" oft skeptisch gegenüber: Einerseits werde das Blatt oft nicht gelesen, andererseits liege das finanzielle Risiko letzten Endes nicht beim Verlag oder Herausgeber, 
sondern bei den Gemeinden. Und im Blick auf zurückgehende Spenden und wachsende andere Aufgaben in der Gemeinde wird gefragt, ob diese Finanzierung der Kirchenzeitung nach Vorrang haben kann.

Der zweite Schwachpunkt ist die wachsende Überalterung der Leser, eine Erscheinung, die in Deutschland schon weiter fortgeschritten ist. In dieser Hinsicht hat die CPA ein neues Konzept entwickelt, um an junge Leser heranzukommen, auf das ich später zurückkomme.

Ich möchte zunächst noch einige Äußerungen zur Situation der US-Kirchenpresse aus Gesprächen refererieren, die bei einem Informationsbesuch des Leiters der Publizistischen Kommission der Deutschen Bischofskonferenz, Dr. Hermann Josef Spital (Trier), im Oktober 1995 in New York mit führenden Medienexperten des Erzbistums geführt werden konnten. Der Leiter der Pressestelle des Erzbistums New York, Joe Twilling, nannte zwei Dinge, die ein Bischof für sein Bistumsblatt tun müsse: "Seine Pfarrer anhalten, den Full coverage-Plan zu erfüllen und soviele Exemplare abzunehmen, daß jeder katholische Haushalt eins bekommt; und seiner Redaktion Freiheit in der Berichterstattung geben."

Die Problematik des Vertriebssystems habe ich bereits angedeutet. Mit der Forderung nach redaktioneller Freiheit sprach Twilling ein Problem an, daß im vergangenen Jahr in der amerikanischen Kirchenpresse wieder stärker in die Diskussion gekommen ist. 1993 hatte die CPA ein Dokument mit Richtlinien über "Freiheit und Verantwortung in der katholischen Presse" verabschiedet, das ausgegangen war von einigen Fällen redaktioneller Zensur durch bischöfliche Herausgeber. Im Prinzip basiert das Dokument auf den Aussagen von "Communio et progressio" und den nachkonziliaren Auffassungen darüber, daß auch kirchliche Presse dem Wesen nach Presse ist. Im vergangenen Jahr flammte die Diskussion neu auf, nachdem der angesehene Theologieprofessor P. Richard McBrien von der Notre Dame Universität in einem Artikel beklagt hatte, daß viele Bistumszeitungen im Gegensatz zu autonomen und freien katholischen akademischen Einrichtungen "zumindest indirekt von der Sicht ihres Herausgebers, nämlich des Ortsbischofs, kontrolliert werden".

Etwa gleichzeitig schlug der Jesuit Thomas C. Widner, der drei Jahre lang vergeblich versucht hatte, einen kompetenten Chefredakteur für die Bistumszeitung von Chicago, "The New World", zu finden, in die gleiche Kerbe. "Katholische Diözesanblätter sind Mittel der Öffentlichkeitsarbeit", schrieb er in einem Artikel mit der Überschrift: "Wohin sind alle unsere Leser verschwunden?". Diözesanblätter, so präzisierte Widner, "sind keine richtigen Zeitungen in dem Sinne, daß sie umfassend über alle Vorgänge in der Kirche berichten".

In einem Kommentar im CPA-Mitteilungsblatt „The Catholic Journalist" entgegegnete der frühere Chefredakteur der Diözesanzeitung 
von New York, Jerry Costello, der jahrzehntelang führend in der CPA wirkte: „Ich habe nie den Anspruch verteidigt, daß Diözesanzeitungen einer säkularen Zeitungen entsprechen müssen; aber ich habe mich immer entschieden dagegen gewehrt, daß sie Mittel der Öffentlichkeitsarbeit sind. Ich siedele sie an zwischen säkularer Presse und Public Relations-Veröffentlichungen mit der Aufgabe, einerseits der kirchlichen Verkündigung $\mathrm{zu}$ dienen, andererseits der offenen Berichterstattung (über die Kirche)." Somit sehen die Redakteure der US-Kirchenpresse sich oft in demselben Dilemma wie ihre Kollegen in der Kirchenpresse anderer Länder. Im "Catholic Journalist" bezeichnete Paul A. Barra es als eine "natürliche Spannung", wenn Bischöfe Herausgeber von Zeitungen sind und Redakteure pressegerecht arbeiten wollen. Zur „Entspannung" schlug Barra zwei Wege vor: entweder einen Redaktionsrat, der zwischen Herausgeber und Redaktion vermittelt, oder einen nicht-kirchlichen Verlag, der unabhängig vom kirchlichen Herausgeber mache. Die generelle Einrichtung eines Beraters oder Ratsgremiums für Konfliktfälle war auch schon in den CPA-Richtlinien über "Freiheit und Verantwortung" von 1993 enthalten.

Besser als solche Gremien findet der Pressesprecher von New York, Joe Twilling, ein offenes Verhalten des Bischofs zu den Medien. Er kann darauf hinweisen, daß dafür der Erzbischof von New York, Kardinal O'Connor, ein Beispiel ist. O'Connor gilt als konservativer Theologe und "Hardliner", aber - so Twilling - "das stört die Medienleute nicht. Für sie ist entscheidend, daß der Kardinal gesprächsoffen ist und sich öffentlich zu Wort meldet." O'Connor ist in New York fast wöchentlich in den Schlagzeilen. Seine Pressesprecher hat engen Kontakt mit den Journalisten der New Yorker Tagespresse ebenso wie mit den Redakteuren der Rundfunk- und Fernsehstationen. Gerade auch in prekären Skandalfällen rät er dem Kardinal, in die Offensive zu gehen. "Ich sage ihm: Wenn wir über einen Vorfall nichts sagen, bringt die Presse ihn groß auf Seite 1, aber wenn wir Stellung beziehen, bringt sie ihn auf Seite 7 unter ,ferner liefen" und muß dazu auch veröffentlichen, welche Meinung wir dazu haben." Als Beispiel nennt Twilling den kürzlichen Tod des Weihbischofes von New York, eines Schwarzen, der angeblich an Aids gestorben war. Um Medienspekulationen zuvorzukommen, stellte der Kardinal sich zum Pressegespräch, dementierte oder bestätigte zwar nicht die Aidsvorwürfe, aber stellte die Persönlichkeit seines Weihbischofes so dar, wie er sie erlebt hatte. Die Folge: Die Aids-Geschichte trat in den Hintergrund.

In die Öffentlichkeit hinein wirkt der New Yorker Kardinal auch durch eine Kolumne, die er regelmäßig in seinem Bistumsblatt veröffentlicht. Darin nimmt er zu aktuellen Fragen Stellung. "Jeden Donnerstagmorgen“, so Twilling, "schickt die New York Times' jemand herüber, der ein Andruckexemplar unserer Kirchenzeitung holt, um den Artikel des Kardinals aktuell auswerten zu können."

In diesem Zusammenhang wies der Pressesprecher generelle Besorgnisse darüber zurück, daß der Kirchenpresse durch die säkularen 
und insbesondere die neuen elektronischen Medien an Bedeutung verlieren werde. Als das Radio aufkam, habe man gesagt: jetzt geht es den Zeitungen schlecht; als das Fernsehen sich ausbreitete, habe man gesagt: jetzt geht es dem Rundfunk schlecht. Jetzt, bei der Ausbreitung der Multimedia, zeige sich: der Rundfunk erlebt eine Wiedergeburt, und die Presse erhält mehr Bedeutung als zuvor. Als Grund dafür gab Twilling an, daß es die Journalisten der Presse sind, die die meisten Nachrichten aufspüren und recherchieren. "Das meiste von dem, was der Rundfunk und das Fernsehen an Nachrichten bringen, greifen sie aus der Presse auf!"

Die Chefredakteurin des "Catholic New York", Ann Buckley, führte als Grund für ihre Überzeugung von der Zukunft der Kirchenpresse an, daß die Menschen immer auch noch lesen wollen, was sie gehört oder gesehen haben, und daß sie darüber noch mehr erfahren wollen, Hintergründe und Folgen kennenlernen wollen. Als Beispiel führte sie den letzten Papstbesuch in New York im Oktober 1995 an. Er wurde von den säkularen Medien in New York im Fernsehen und Rundfunk in einem Ausmaß übertragen wie bei keinem anderen Ereignis der letzten Zeit. "Und doch wollten viele Leute später nachlesen, was er gesagt hat und was andere über den Besuch meinen", sagte Ann. Von ihrer Kirchenzeitungsausgabe mit den Wortlauten der Papstansprachen mußten Nachdrucke erstellt werden.

Das alles sind persönliche Schlaglichter auf Einzelaspekte in der katholischen Presse der USA. Doch auch generell sehen die Redakteure, Verleger und Herausgeber der Bistumspresse für die Zukunft konkrete Chancen. Dabei wissen sie allerdings, daß zur Verwirklichung viel mehr als bisher getan werden muß. Grundlage für die Zukunftshoffnungen sind dabei Entwicklungen in der Rolle der katholischen Kirche in der amerikanischen Gesellschaft, eine Rolle, die mit der Position der Kirche in den europäischen Ländern nicht verglichen werden kann. Die katholische Kirche in den USA ist längst aus einer MinderheitenPosition zu einer mitbestimmenden Gesellschaftskraft geworden. In einer Zeit, in der die Gesellschaft in Amerika in ihren demokratischen und freiheitlichen Grundzügen ebenso erschüttert wird wie durch soziale, wirtschaftliche und wissenschaftliche Umbrüche, kommt dem Wertesystem der katholischen Kirche wachsende Bedeutung zu. Insbesondere die Haltung der Bischöfe zu Krieg und Abrüstung, zu Armut und Ungleichheit der Rassen, zum Schutz des ungeborenen und des alten Lebens scheinen der Gesellschaft Fixpunkte zu geben, die sie dringend sucht. Hinzu kommt, daß der amerikanische Katholizismus selbst kraftvoll und stark in den Menschen verankert ist, - oft zwar in traditionellen Formen, aber nicht so auf dem Rückzug oder zurückgedrängt wie in Europa. Die "Stimme der Kirche" spricht in der amerikanischen Öffentlichkeit mit und wird gehört.

Diese Ausgangssituation bietet der Bistumspresse neue Chancen. Gewiß auch aus Wahlkalkül, aber dennoch aufschlußreich war es, daß 
US-Präsident Clinton Ende vergangenen Jahres zum erstenmal eine Gruppe von Redakteuren der katholischen Presse exklusiv im Weißen Haus empfangen hat und ein längeres Informationsgespräch mit ihnen führte.

Nachdem viele Bistumszeitungen in den letzten Jahren ihre Redaktionen professionalisiert, mit neuen Techniken ausgestattet und ihr Image durche neue Layouts aufgebessert haben, verfolgt die CPA jetzt in einem Long range-Plan, einem Langfristplan, mehrere Ziele zur Verbesserung der Anzeigen- und Leserwerbung. Der geschäftstüchtige Direktor der CPA, Owen McGovern, der z.B. auch neue Quellen des Sponsoring für die Konferenzen der Verleger und Redakteure erschloß, faßt sein ceterum censeo zusammen in der Forderung: „Promotion, promotion, promotion."

Vor zwei Jahren führte die CPA für die Leser der USKirchenpresse erstmals eine Datenerhebung über ihre Leser durch. Diese ergab, daß die Katholiken weitaus bessere Ansprechpartner für die Werbung sind als die Durchschnittsbevölkerung. Während nur $40 \%$ der Durchschnittsamerikaner eine College-Ausbildung besitzen, sind es $66 \%$ bei den Katholiken. Während $64 \%$ der Durchschnittsbevölkerung ein Haus besitzen, sind es $80 \%$ der Katholiken. Während zur Gruppe mit einem Durchschnittseinkommen 32\% der Bevölkerung zählen, sind es bei den Katholiken $38 \%$, und während $17 \%$ der Amerikaner über höheres Einkommen (was in den USA bei 100.000,-- DM im Jahr beginnt) verfügen, sind es bei den Katholiken 23\%. Mit diesen Daten erstellte die CPA 1995 eine Werbemappe mit dem Titel „Entdecke das ungenutzte Potential der amerikanischen Katholiken".

Zugleich mit der Veröffentlichung dieser Mappe gründete die CPA eine eigene Werbeagentur, das Catholic Advertising Network (CAN), das nationenweit Anzeigen für die Mitgliedszeitschriften akquirieren soll. Bisher betrieben die Zeitungen nur ihr eigenes Anzeigengeschäft, jetzt versucht die CPA, einen gemeinsamen Anzeigentarif zu erarbeiten.

Der zweite Schwerpunkt in der Promotion liegt bei der Leserwerbung. Die CPA hat hierfür eine Reihe von Vorschlägen für die einzelnen Bistumsblätter erarbeitet. So haben im vergangenen Jahr mehrere Bistumszeitungen bei bestimmten Anlässen - Bischofstag, Pressemonat ihre Auflagen auf die Gesamtzahl der katholischen Haushalte erhöht und den Nichtbeziehern durch die Gemeinden eine zeitlang kostenlos Exemplare zustellen lassen. Bei anschließenden Abo-Besuchen oder Anschreiben konnten die verkauften Auflagen in den meisten Fällen um rd. $10 \%$ erhöht werden.

Nicht für den Augenblick, wohl aber auf Zukunft gesehen verspricht Owen McGovern sich viel vom jüngsten Projekt der CPA: „Newspapers in Education". Diese Projekt "Zeitung in der Schule“, das 
in Deutschland vom Aachener IZOP-Institut seit über zehn Jahren mit wachsendem Erfolg in der säkularen Presse durchgeführt wird, ist in den USA im vergangenen Jahr von der CPA zusammen mit einer Organisation SMART für Schulfinanzen entwickelt worden. Die Organisation dient der Entlastung der katholischen Privatschulen bei der Erhebung der Schulgelder und sponsort verschiedene Schulhilfen, darunter auch drei Lehrer- und Erziehungszeitschriften der Nationalen Katholischen Erziehungs-Vereinigung (NCEA).

Angeregt durch Projekte des "Wallstreet Journals" und der Tageszeitung "US Today", entwickelte die CPA mit SMART ein Curriculum für den Gebrauch von Diözesanzeitungen im Schulunterricht. Der Lehrplan ist gegliedert in Material für die Elementary und die Secondary School, die Grund- und Mittelschulen also. Es kann in einem Zeitraum von 12 oder 24 Wochen eingesetzt werden und baut jeweils in sieben Stufen das Wissen über eine Zeitung auf. Endziel ist die Erstellung einer eigenen Zeitung durch die Schüler.

Das Programm soll durch die Bistumszeitungen an die örtlichen katholischen Schulen herangetragen werden. Sie können dafür entweder Überdruck-Exemplare verschicken oder von Schulen, Schülern und/ oder Eltern ermäßigte Bezugspreise nehmen, wobei McGovern davon ausgeht, daß nicht jeder Schüler während des Unterrichts ein Exemplar braucht, sondern jede Klasse nur eine kleine Zahl.

„Wenn man bedenkt, daß viele Schulkinder heute gar keine Kirchenzeitung kennen", sagt der Präsident von SMART, Sam Rosen, "bietet das Programm die beste Möglichkeit, Kindern im Unterricht damit bekanntzumachen." Owen McGovern von der CPA sieht einen doppelten Effekt: „Durch die Beschäftigung mit dem Inhalt der Zeitung wird das katholische Wissen verstärkt, und das Programm kann uns die Kirchenzeitungsleser der nächsten Generation sichern. Die Schüler von heute sind unsere Bezieher von morgen!"

Einen dritten Weg zur Promition der Bistumszeitungen will die CPA im Internet erschließen. Sie gründete eine ad hoc-Kommission "On-line", die in zwei große Richtungen überlegt: ein "In-houseNetwork" zu errichten, also eine On-line-Kommunikation für Kirchenzeitungen untereinander, und einen Kommunikationsweg nach draußen. Nach draußen sind im vergangenen Jahr inzwischen schon die ersten US-Kirchenzeitungen gegangen. Als erste machte die Kirchenzeitung der Diözese Raleigh, Nord-Karolina, "NC Catholic", aus der Not eine Tugend. Sie mußte aus finanziellen Gründen vom einwöchigen auf zweiwöchiges Erscheinen umgestellt werden. Um einen neuen Zugang zu diözesanen Informationen zu erschließen, fragte der stellvertretende Chefredakteur Matt Doyle seine Bistumsverantwortlichen: "Was haltet ihr von einer ,24 Stunden am Tag, sieben Tage in der WocheKirchenzeitung"?" Zusammen mit dem Diözesandirektor für Computerdienste, Tod Wall, entwickelte die Redaktion ihre erste Home page und 
stellte ihr erstes Programm fürs World Wide Web zusammen. Im Angebot sind nicht nur neue Nachrichten und Artikel aus der Kirchenzeitung, Gottesdienst- und Gemeindeveranstaltungen sowie ein Führer durch die Fernsehprogramme, sondern auch Stellenangebote für den Arbeitsmarkt.

Inzwischen haben weitere Bistumszeitungen den Weg ins Internet beschritten, z.B. der "Criterion" in Indianapolis. Die Informationen werden kostenlos angeboten (wie von den säkularen Zeitungen auch, die Zusammenfassungen oder Ausschnitte ihres Inhaltes kostenlos anbieten und diesen Dienst als Werbung betrachten). „Zuerst haben wir gedacht, ohne Gebühren könnten wir nicht viel machen", stellte der Direktor der Computerdienste, Tod Wall, fest, „aber durch Zusammenarbeit mit unseren (katholischen) Universitäten und anderen Anbietern ist es überraschend, mit wie wenig Geld wir auskommen. Es ist jedenfalls viel billiger als die Produktion eines Fernsehspots."

Owen McGovern warnt bei allen diesen Projekten davor, auf schnelle Erfolge zu hoffen. Er betrachtet sie als Investitionen in die Zukunft.

Ferdinand Oertel, Aachen 


\section{Zur Lage der evangelischen Kirchengebietspresse}

Die evangelische Kirchengebietspresse befindet sich in der Krise. Dieses Lamento wird seit Anfang der achtziger Jahre immer dann angestimmt, wenn die Rede auf die (seit der Vereinigung) 16 Kirchengebietsblätter kommt. Die Gesamtauflage von knapp 700.000 Exemplaren (bei 29 Millionen Protestanten) hört sich zwar noch immer gewaltig an. Sie täuscht jedoch darüber hinweg, daß die meisten Publikationen aus dieser Kategorie Einbußen in ihrer Auflage von bis zu fünfzig Prozent in den letzten zwanzig Jahren haben hinnehmen müssen.

Und dennoch: Obwohl krisengeschüttelt, sind die Blätter alles in allem stabil. Das hängt zum einen damit zusammen, daß die evangelischen Landeskirchen ihren Zuschußverpflichtungen nachkommen. Zum anderen hat sich der Zusammenschluß der evangelischen Kirchengebeits- und katholischen Bistumszeitungen in der Agentur "Konpress" bewährt. Der Ertrag aus dem Anzeigengeschäft hat sich innerhalb der letzten zehn Jahre beträchtlich steigern lassen. Zu einer gewissen Stabilität hat auch eine Professionalisierung in den Redaktionsstuben der einzelnen Publikationen beigetragen. Moderne Layoutkonzepte trugen ebenso dazu bei, daß der Leserschwund bei einzelnen Blättern aufgehalten werden konnte und ein Auflagenplus in bescheidenem Umfang zu verzeichnen ist.

$\mathrm{Ob}$ damit der Abwärtstrend insgesamt aufgehalten oder sogar umgekehrt werden kann? In der Branche selbst bestehen Zweifel. Der inhaltliche Auftrag läßt sich bei weitem nicht mehr so präzise fassen wie unmittelbar nach dem Zweiten Weltkrieg, als die Kirchen in den Besatzungszonen mit die ersten waren, die Lizenzen für den Zeitungsdruck bekamen. Wer sich über kirchliche und weltliche Ereignisse informieren wollte, kam um diese Erzeugnisse nicht herum. Ähnlich war es in der zweiten Hälfte der achtziger Jahre in der DDR. Dort stellten die evangelischen Kirchengebietsblätter eine Ersatzplattform dar, um sich aus einem unideologischen Blickwinkel - mehr über politische und gesellschaftliche als über kirchliche Vorgänge - informieren zu lassen. Nach der Wende ließ das Interesse an den Kirchenblättern schlagartig nach. Sie hatten ihren Auftrag erfüllt.

Warum soll ein evangelischer Christ das Kirchengebietsblatt seiner Landeskirche abonnieren? Was bietet es ihm, das er nicht an anderer Stelle ebenso bekommt? Hauptkonkurrent Nummer eins der Kirchenzeitungen sind die Gemeindebriefe. Der Grad an Professionalisierung wurde in den vergangenen zwei Jahrzehnten kräftig erhöht. Dies sah das Konzept des Gemeinschaftswerkes der Evangelischen Publizistik (GEP) in Frankfurt am Main vor. Und es hat sich umsetzen lassen. Wer über örtliche und bedingt auch über regionale kirchliche 
Vorgänge informiert sein will, für den ist der Gemeindebrief das wichtigste Medium. Es hat unter allen kirchlichen Druckerzeugnissen die höchste Einschaltquote. Nach wie vor wird kirchlichen Ereignissen auch in der (säkularen) Lokalpresse viel Raum gewährt. Der Wochenrhythmus der Kirchengebietsblätter kann im Blick auf die Aktualität nicht mit der lokalen und regionalen Presse mithalten. Regionalseiten und -beilagen in den Kirchengebietsblättern müssen um das "deja vu“-Erlebnis des Lesers („Das kenne ich schon aus der Lokalzeitung“) beträchtliche Anstrengungen unternehmen, damit ihr Angebot nicht wie ein zweiter Aufguß erscheint.

Ganz auf lokale und regionale Berichterstattung verzichtet die neue Publikation der Evangelischen Landeskirche in Baden. Sie hat Ende März 1996 ihre Wochenzeitung "Aufbruch" eingestellt, die durch das farbige Monatsmagazin "Standpunkte" ersetzt werden soll. Mit der neuen Zeitschrift wolle man "weite Kreise" der badischen Landeskirche ansprechen. "Die Macher verstehen das Magazin", so heißt es in der Ankündigung des Evangelischen Presseverbandes für Baden, "als Orientierungshilfe in Gesellschaft, Leben und Glauben." Anders als die klassischen Kirchenzeitungen stelle "Standpunkte" nicht Kirche und Glaube in den Mittelpunkt, sondern wolle mit den Lesern über lebensnahe Themen ins Gespräch kommen. Thematisiert werde nicht der Glaube, sondern was er bewirken könne. Nicht ausdrücklich erwähnt, aber vom Konzept her sicher gewollt: Das neue Layout soll dazu führen, auch junge Leute anzusprechen, ob innerhalb oder außerhalb der Kirche.

Eine erste Auswertung von Leserreaktionen in Baden hat ergeben, $\mathrm{da} B$ Aufmachung und Inhalt zwar überzeugen, aber der Wurzelgrund der eigenen Landeskirche nur noch bruchstückhaft wahrzunehmen sei. Der dauerhafte Verzicht auf eine lokale und regionale Berichterstattung über kirchliches Leben könnte dazu führen, daß "Standpunkte" in allen evangelischen Landeskirchen Deutschlands angeboten wird. Und dies nicht nur aus wirtschaftlichen Erwägungen, sondern auch, weil es ein evangelisches Monatsblatt mit Magazincharakter bislang nicht gibt. Sowohl die "Evangelischen Kommentare" als auch die "Lutherischen Monatshefte", die bislang die Brücke zwischen kirchlichen Insidern und an religiösen Themen interessierten Zeitgenossen zu schlagen versuchte, unterscheiden sich in ihrem Konzept von dem der "Standpunkte".

Es gibt auch Stimmen in der evangelischen Publizistik, die davor warnen, den lokalen und regionalen Bezug der Kirchengebietsblätter zu streichen. Zur Begründung führen sie an, daß der Stellenwert der kirchlichen Berichterstattung in der säkularen Presse keineswegs gesichert ist. Nicht nur, daß rund zwei Drittel aller Medienleute nicht mehr Kirchenmitglieder sind, sondern daß vor allem der Stellenwert von Glaube und Kirche in der Gesellschaft zunehmend umstritten sei. Hinzu kommen vereinzelt in der überregionalen Presse anzutreffende kirchenfeindlichen Beiträge. Vor diesem Hintergrund, so heißt es, dürfe die Bedeutung der Kirchengebietsblätter nicht unterschätzt werden. 
Freilich unternehmen die evangelischen Landeskirchen landauf landab zahlreiche Anstrengungen, die Wichtigkeit dieser Wochenzeitungen in Zweifel zu ziehen. Es mehren sich die Stimmen, man müsse Mitgliederpublikationen - kostenlos - allen evangelischen Haushalten zustellen, um auch die zu informieren, die kein Kirchengebietsblatt lesen, aber dennoch über landeskirchliche Themen informiert sein sollten. Diese Zielgruppe zu erreichen, ist ein berechtigter Wunsch. Aber er muß in ein Konzept evangelischer Publizistik eingebunden sein, um entsprechende Wirkung erzielen zu können.

Eine Ausnahmesituation in positiver Hinsicht scheint noch immer in Bayern zu bestehen. Dort gibt es seit 1884 das "Evangelische Sonntagsblatt aus Bayern ", das in einem Privatverlag in Rothenburg ob der Tauber erscheint. Nach dem Krieg wurde - sozusagen als offizielles Blatt der Landeskirche - das "Sonntagsblatt - Evangelische Wochenzeitung für Bayern" in München gegründet. Während das Münchener Sonnagsblatt mit modernem Layout und mehreren Regionalausgaben Ende 1995 eine verkaufte Auflage von fast 55.000 Exemplaren erreichte, erscheint die Rothenburger Konkurrenz seit 1884 mit einem Holzschnitt aus der Ludwig-Richter-Schule und einer Andacht auf der Titelseite, was auf den ersten Blick etwas antiquiert erscheint. Mit Ende letzten Jahres fast 38.000 verkauften Exemplaren, erfreut sich das Rothenburger Sonntagsblatt großer Beliebtheit in der Landeskirche. Zum Herausgeberkreis gehören namhafte Protestanten, und Chefredakteur Günter Saalfrank ist ein vom Gemeindedienst freigestellter Pfarrer, der zudem über eine journalistische Ausbildung verfügt.

Das nostalgisch anmutende Layout sei Programm, sagt Günter Saalfrank über das Rothenburger Blatt. Themen des Glaubens zeichneten das "klare Profil" aus. Neben Informationen über das kirchliche Leben liege der Schwerpunkt auf geistlichen Beiträgen, Andachten, Meditationen. Daß die bayerische Landeskirche als einzige innerhalb der EKD zwei Kirchenzeitungen fördere, die sich im Grunde keine Konkurrenz machen, deutet Saalfrank als ein Ja zur Volkskirche mit ihren unterschiedlichen Strömungen. Die Bilanz gebe der Kirche recht, so der Chefredakteur des Rothenburger Sonntagsblattes, denn mit beiden Blättern würden rund zehn Prozent der 2,2 Millionen Evangelischen im Freistaat erreicht. Eine Quote, die in keiner anderen Landeskirche anzutreffen ist.

Die Krisenerscheinungen der evangelischen Kirchengebietsblätter lassen sich nicht wegdiskutieren. Die Ruhepause angesichts einer relativen wirtschaftlichen Stabilität sollte genutzt werden, um konzeptionell dieser Wochenpublizistik zum Überleben zu verhelfen. Würde das eine oder andere Blatt eines Tages eingestellt werden, dann wäre die Hoffnung auf ein Wiedererscheinen zu einem späteren Zeitpunkt wohl unrealistisch. Im Augenblick bleibt es erst einmal dabei, daß Totgesagte länger leben. 


\section{Medien, Religion und Kultur}

Internationales und interdisziplinäres Boulder-Forum 1996

„Medien, Religion, Kultur" war das Thema einer als „erste interdisziplinär und international" angekündigten Konferenz, die vom 11. bis 14.01.1996 an der Universität von Colorado in Boulder (USA) veranstaltet wurde. Das Thema traf offensichtlich auf großes Interesse, denn über 200 Teilnehmer fanden für sich diese Tage unter winterlicher Sonne in Boulder, unweit von Denver, auf dem Campus der Universität ein.

Die ersten Vollversammlungen beschäftigten sich mit kurzen Beiträgen aus Indien, Südafrika und Peru mit den internationalen, interkulturellen und interreligiösen Perspektiven des Konferenzthemas. Die meisten anderen Vollversammlungen allerdings, einschließlich des Hauptvortrages von Martin Marty (Chicago) "Constructing Religion in the Media", waren mehr den inneramerikanischen Erfahrungen gewidmet: "Civil Religion" - ,Blutopfer und die Nation" - (Carolyn Marvin), "Visual Text. Sacred Image and Scripture in modern Media" (verschiedene Autoren) waren Themen solcher Vollversammlungen.

In einer Fülle verschiedener ,Panels` kamen eine Anzahl von Konferenzteilnehmern mit ihren Forschungen, Erfahrungen und Überlegungen $\mathrm{zu}$ Wort. Insgesamt 27 solcher Arbeitssitzungen mit jeweils drei Autoren wurden angeboten, was immerhin etwas über 70 verschiedene Beiträge bedeutet. Für jeden Teilnehmer einer so ausgestatteten Konferenz mit einer solchen Fülle von Angeboten ist es natürlich schwierig, die Übersicht zu behalten oder gar Trends festzustellen. Einige Themengruppen aber geben vielleicht einen Eindruck von den angesprochenen Erfahrungen und Überlegungen. Religion in einer elektronischen Welt war ein wiederkehrendes Thema verschiedener Präsentationen, die Darstellung der Religion in verschiedenen Medien war ein anderes, ebenso wie Religion und Technologie, Religion und Medien in der Öffentlichkeit. Medienpraxis verschiedener kirchlicher Gruppen, aber auch Medienstudien und die Rolle der Medienempfänger standen im Mittelpunkt anderer Arbeitsgruppen. Auffallend war das in der einen oder anderen Form immer wiederkehrende Thema einer ,Visualisierung' der Religion und auch der biblischen Botschaft. Für die Ausbildung zukünftiger Priester und Pastoren wurde in einer Arbeitsgruppe eine Re-Interpretation der christlichen Botschaft im Lichte der modernen elektronischen Kultur gefordert: Bisher waren Bücher, Bibliotheken und geschriebene Thesen die Grundlage theologischer Ausbildung. Jetzt sollten es moderne Video- und Audio-,Texte' sein. Der Theologe habe die elektronische Kultur ernst zu nehmen, die durch das Narrative, Teamarbeit und internationale Vernetzung (Inter-

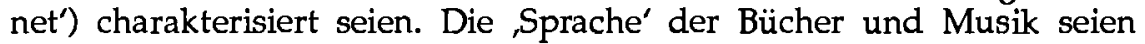
Zeichen unserer Zeit. 
Clifford Christians hatte mehrfach zusammen mit anderen Gelegenheit, auf die Bedeutung einer Medienethik hinzuweisen. Eine solche Ethik wächst aus der Existenz und Verpflichtung der Wahrheit. Können wir daraus moralische Universalregeln entwickeln? Eine reine ,Aufklärungsmoralität ' ist wohl nicht mehr ausreichend. Offensichtlich gibt es "Proto-Normen", wie etwa die Heiligkeit, Unantastbarkeit und Würde menschlichen Lebens.

Die Konferenz in Boulder hatte zwar nicht im Namen, aber in Wirklichkeit einen stark christlich-kirchlichen Charakter. Viele Vertreter christlicher Kirchen und Denominationen oder kirchlich gebundene Professoren waren anwesend, und manchmal hatte man den Eindruck, mehr an einer innerkirchlichen Diskussion als einer akademischen interkulturellen Veranstaltung teilzunehmen. Andere Religionen wie Islam, Buddhismus, Hinduismus und deren Beziehung zu den Medien oder generell zur Kommunikation kamen im Gesamt der Veranstaltung kaum zur Sprache.

Auch hätte man sich unter dem Stichwort ,Medien' eine größere Bandbreite gewünscht, wo eigentlich der Gesamtbereich der sozialen Kommunikation hätte angesprochen werden müssen. "Civil Religion" etwa drückt sich eben nicht nur in Massenmedien aus und die öffentliche Meinung wird nicht nur durch die Massenmedien oder die elektronische Welt allein bestimmt.

Das Boulder-Kommunikationsforum war weithin in Konzeption und Teilnehmerschaft eine nordamerikanische Veranstaltung, bei der man sich halt mit ,hausinternen' Erfahrungen, Befürchtungen und Erwartungen beschäftigte. Aus Norwegen und Südafrika waren kleinere Teilnehmergruppen anwesend, die über gemeinsame Erfahrungen im neuen Südafrika hinaus aber kaum das Gesamtbild einer nordamerikanischen Veranstaltung änderten. Dabei wäre wahrscheinlich eine stärkere Beachtung von ,Dritte Welt'-Erfahrungen und der Rolle von Religion und Kommunikation in kulturanthropologischer Sicht eine große Bereicherung auch für die Teilnehmer aus den USA gewesen, die nicht leicht über den eigenen Rahmen hinauszublicken scheinen.

Es ist das Verdienst von Steward Hoover und seinem Team von der Universität von Colorado, mit viel Mühen ein solches Forum veranstaltet zu haben. Trotz verschiedenster Herkunft der Teilnehmer gab es vom ersten Tag an das Gefühl eines gemeinsamen Anliegens, was auch durch die überrraschend hohe Zahl der Teilnehmer belegt wurde, die trotz starker Schneefälle im Osten des Landes den Weg in die winterliche Sonne Boulder's fanden.

Mit Spannung darf man auf eine Fortsetzung dieser Foren oder ähnlicher Veranstaltungen warten, die auch dem Anliegen einzelner Fachgruppen zugute kommen. So gab Boulder der "Association for Communication in Theological Eduacation" (ACTE) Gelegenheit, sich 


\section{BERICHTE}

zum zweiten Mal seit der Gründung an der Yale School of Divinity im Jahre $1992 \mathrm{zu}$ treffen. In der bisher von William Fore, dem früheren Kommunikationsdirektor des Nationalen Kirchenrates (NCC) der USA, geleiteten Vereinigung arbeiten Professoren zusammen, die an theologischen Hochschulen im Fach Kommunikation unterrichten. Von anfänglich 30 Mitgliedern hat sich zumindest die Bezieherschaft des von der ACTE zweimal jährlich veröffentlichten Nachrichtenblattes "Connections“ (William Fore, 19 Highview Road, Madison, CT 06443) auf 140 erhöht. Mitglieder der Vereinigung planen mit Unterstützung des Nationalen Kirchenrates ein Kommunikationsinstitut, das Studenten theologischer Hochschulen in ihren Studien im elektronischen Zeitalter begleiten soll. Wegen relativ zahlreicher theologischer Schulen in Chicago ist eine solche ökumenisch konzipierte Einrichtung in dieser Stadt vorgesehen.

Eine Veröffentlichung der Überlegungen und wichtigerer Beiträge der Konferenz von Boulder wäre sicher wünschenswert, um auch jene zu beteiligen, die in Boulder nicht dabei sein konnten. Es würde auch helfen, nordamerikanische Erfahrungen anderen Ländern und Kontinenten zugänglich zu machen und so ein breiteres internationales Gespräch über Medien, Religion und Kultur zu fördern.

Franz-Josef Eilers, Tagaytay City (Philippinen) 


\title{
„Die Medien: Forum der Gegenwart zur Förderung
} der Rolle der Frau in der Gesellschaft"

\author{
Botschaft Johannes Pauls II. zum 30. Welttag der Sozialen
} Kommunikationsmittel 1996

\section{Liebe Brüder und Schwestern!}

Das diesjährige Thema für den Welttag der Sozialen Kommunikationsmittel „Die Medien: Forum der Gegenwart zur Förderung der Rolle der Frau in der Gesellschaft" unterstreicht, daß nicht nur bei der Förderung von Gerechtigkeit und Gleichsteliung für die Frauen, sondern auch bei der Förderung der Anerkennung für ihre spezifischen weiblichen Gaben, die ich an anderer Stellen den "Genius der Frau" genannt habe (vgl. Mulieris dignitatem, 30; Brief an die Frauen, 10), die Medien eine entscheidende Rolle spielen.

Im vergangenen Jahr versuchte ich in meinem Brief an die Frauen vor allem mit den Frauen selber einen Dialog darüber in Gang zu bringen, was es heißt, heutzutage Frau zu sein (vgl. Nr. 1). Ich wies auch auf einige Hindernisse hin, "die in vielen Teilen der Welt den Frauen noch immer die volle Einbeziehung in das gesellschaftliche, politische und wirtschaftliche Leben verwehren" (Nr. 4). Diesen Dialog können die Medien heute pflegen und unterstützen, ja sie sind direkt dazu verpflichtet. Medienleute werden so, und das ist lobenswert, oft zu Fürsprechern derer, die keine Stimme haben und am Rande der Gesellschaft leben. Sie befinden sich der einzigartigen Lage, im Hinblick auf zwei ernste Probleme bezüglich der Frauen in der heutigen Welt auch das Bewußtsein aufzurütteln.

Das erste ist, wie ich in meinem Brief bemerkte, daß die Mutterschaft oft eher bestraft als belohnt wird, obwohl die Menschheit ihr eigenes Überleben jenen Frauen verdankt, die sich zu einem Dasein als Ehefrau und Mutter entschieden haben (vgl. Nr. 4). Es ist sicher eine Ungerechtigkeit, daß solche Frauen in wirtschaftlicher und sozialer Hinsicht deswegen diskriminiert werden sollen, weil sie jener Grundberufung folgten. Ebenso wies ich darauf hin, daß es dringend geboten ist, überall die tatsächliche Gleichheit zu erreichen: "gleichen Lohn für gleiche Arbeit, Schutz der berufstätigen Mutter, gerechtes Vorankommen in der Berufslaufbahn, Gleichheit der Eheleute im Familienrecht und Anerkennung von allem, was mit den Rechten und Pflichten des Staatsbürgers in der Demokratie zusammenhängt" (ebd., Nr. 4).

Zweitens ist die Förderung einer echten Emanzipation der Frau ein Akt der Gerechtigkeit, über den man nicht länger hinwegsehen kann; es handelt sich dabei auch um eine Frage zum Wohl der Gesellschaft. Glücklicherweise wächst das Bewußtsein dafür, daß es 
Frauen ermöglicht werden muß, bei der Lösung der ernsten Probleme der Gesellschaft und der Zukunft der Gesellschaft eine aktive Rolle zu spielen. In allen Bereichen „wird sich eine stärkere soziale Präsenz der Frau als wertvoll erweisen, denn sie wird dazu beitragen, die Widersprüche einer Gesellschaft herauszustellen, die auf bloßen Kriterien der Leistung und Produktivität aufgebaut ist, und sie wird auf eine Neufassung der Systeme dringen zum großen Vorteil der Humanisierungsprozesse, worin sich der Rahmen für die "Zivilisation der Liebe“ abzeichnet" (ebd., Nr 4).

Die "Zivilisation der Liebe" besteht ganz besonders in einer radikalen Anerkennung des Wertes des Lebens und des Wertes der Liebe. In beiden Bereichen ist die Frau besonders qualifiziert und privilegiert. Was das Leben angeht, so erfreuen sich die Frauen, obwohl sie nicht allein für die Achtung des ihm innewohnenenden Wertes verantwortlich sind, wegen ihrer innigen Verbundenheit mit dem Geheimnis der Weitergabe des Lebens einer einzigartigen Fähigkeit zu solchem Handeln. Was die Liebe betrifft, so können Frauen in jede Lebenslage, einschließlich der höchsten Ebenen der Entscheidungsfindung und Beschlußfassung, jene wesentliche weibliche Eigenschaft einbringen, die in der Objektivität des Urteils besteht, das gemildert wird durch die Fähigkeit zum tiefen Verständnis für das Verlangen nach zwischenmenschlichen Beziehungen.

Die Medien, zu denen Presse, Kino, Radio und Fernsehen, die Musikindustrie und Computer-Netzwerke gehören, repräsentieren das Forum der Gegenwart, wo Informationen empfangen und schnell an ein Publikum in der ganzen Welt weitergegeben werden, wo Ideen ausgetauscht, Haltungen entwickelt werden - und wo in der Tat eine neue Kultur Gestalt annimmt. Die Medien sind daher dazu ausersehen, einen mächtigen Einfluß bei der Entscheidung auszuüben, ob eine Gesellschaft nicht nur die Rechte, sondern auch die besonderen Gaben der Frau voll anerkennt und zu schätzen weiß.

Trotzdem sehen wir leider in den Medien häufig nicht die Hochachtung vor den Frauen, sondern ihre Ausbeutung. Wie oft werden sie nicht als Personen mit einer unverletzbaren Würde behandelt, sondern als Objekte, deren Zweck darin besteht, die Vergnügungslust und Machtgier anderer zu befriedigen? Wie oft wird die Rolle der Frau im Geschäfts- und Berufsleben als eine Karikatur des Mannes gezeichnet, wobei die spezfischen Gaben weiblicher Einsicht, ihres Mitleids und Verständnisses, die so sehr zur "Zivilisation der Liebe" beitragen, geleugnet werden?

Die Frauen können selber viel für eine bessere Behandlung der Frauen in den Medien tun, indem sie Erziehungsprogramme in den audio-visuellen Medien fördern, andere, besonders Familien, zu einem kritischen Konsumverhalten auf dem Medienmarkt anhalten, Produktionsgesellschaften, Publizisten, Rundfunk- und Fernsehsender 
und Inserenten mit ihren Ansichten über Programme und Publikationen bekanntmachen, welche die Würde der Frau beleidigen oder ihre Rolle in der Gesellschaft herabsetzen. Außerdem können und sollten sich Frauen selbst für verantwortungsvolle und kreative Positionen in den Medien bereit machen, und zwar nicht im Konflikt mit oder in Nachahmung von männlichen Rollen, sondern indem sie ihrer Arbeit und beruflichen Tätigkeit ihren eigenen "Genius" aufprägen.

Die Medien würden gut daran tun, sich auf die wahren Heldinnen der Gesellschaft, einschließlich der heiligmäßigen Frauen aus der christlichen Überlieferung, als Rollenmodelle für die Jugend und für zukünftige Generationen einzustellen. Vergessen können wir in diesem Zusammenhang auch nicht die vielen Ordensfrauen, die alles aufgeopfert haben, um Jesus zu folgen und sich dem Gebet und dem Dienst an den Armen, den Kranken, den Analphabeten, den Jugendlichen, den Alten und den Behinderten zu widmen. Einige dieser Frauen sind selbst in den Medien tätig - sie arbeiten dafür, daß „den Armen eine gute Nachricht gebracht wird“ (vgl. Lk 4,18).

"Meine Seele preist die Größe des Herrn“ (Lk 1,46). Mit diesen Worten antwortete die selige Jungfrau Maria auf die Begrüßung durch ihre Cousine Elisabeth und anerkannte damit das "Große", das Gott an ihr getan hatte. Das Bild der Frau, das die Medien vermitteln, sollte die Anerkennung einschließen, daß jede weibliche Gabe die Größe des Herrn verkündet, des Herrn, der Leben und Liebe, Güte und Gnade mitgeteilt hat, des Herrn, der die Quelle der Würde und Gleichheit der Frau und ihres besonderen "Genius" ist.

Mein Gebet an diesem 30. Welttag der Sozialen Kommunikationsmittel wird alle, die in den Medien tätig sind, besonders die Söhne und Töchter der Kirche, dazu ermutigen, die echte Förderung der Würde und Rechte der Frauen dadurch voranzubringen, daß sie ein wahres und respektvolles Bild ihrer Rolle in der Gesellschaft projizieren und "die volle Wahrheit über die Frau" zutage treten lassen (Brief an die Frauen, Nr. 12).

Aus dem Vatikan, am 24. Januar 1996, Fest des hl. Franz von Sales.

Johannes Paulus PP II. Aus: L'Osservatore Romano, deutsche Ausgabe vom 2. Februar 1996 